\title{
Spontaneous Gall Bladder Perforation: A rare clinical entity, a diagnostic and surgical challenge
}

\author{
Swapnil Sen ${ }^{1 *}$ and Ujjwal Kumar Parui ${ }^{2}$ \\ ${ }^{1}$ Department of Surgery, BR Singh Hospital \& Research Centre, Eastern Railway, Sealdah , Kolkata West Bengal, India \\ ${ }^{2}$ Department of Surgery, Kachrapara Railway Hospital, Eastern Railway, Kachrapara, West Bengal, India
}

*Corresponding author: Swapnil Sen, Department of Surgery, BR Singh Hospital \& Research Centre, Eastern Railway, Sealdah, Kolkata West Bengal, India

Submission: November 01, 2017; Published: January 03, 2018

Keywords: Acalculus Cholecystitis, Gall bladder perforation, Biliary Peritonitis

\section{Introduction}

Gallbladder perforation requiring an emergent treatment is usually a complication of cholecystitis [1]. Acute cholecystitis develops in up to $2 \%$ of patients affected by asymptomatic cholelithiasis. Gallbladder perforation occurs in 2 to $11 \%$ of acute cholecystitis cases. Due to the high mortality that can be caused by a delay in the correct diagnosis and following adequate surgical treatment, gallbladder perforation represents a special diagnostic and surgical challenge [2].

According to Niemeier (1934), perforations are classified into three categories: type I includes patients with free perforation into the peritoneal cavity, type II describes patients with localized perforation and type III patients with cholecysto-enteric fistulas. Less frequent forms include cholecystobiliary fistula and more complex fistula formations [3]. Cases of intrahepatic perforation of the gallbladder with liver abscess and cholecystohepatic communication have also been reported [4].

Cases reported as idiopathic or as spontaneous gallbladder perforation are not only rare but also have features that are different from those occurring as a complication of cholecystitis. Their different features can be described as peritonitis caused by gallbladder perforation lacking the clinical manifestations, radiological and histopathological characteristics of cholecystitis or gallbladder perforation. As a result, diagnosis is often delayed or even missed [5,6].

\section{Materials and Methods}

We report a single case of Acalculus Cholecystitis with spontaneous gall bladder perforation at a tertiary care apex institution of Eastern India.

\section{Case Report}

A 51 old male patient admitted with complaints of sudden onset pain abdomen mainly over upper abdomen with abdominal distension, single episode of vomiting associated with nausea. Pain was dull aching in nature radiating to the back in the interscapular area. Patient is a known alcoholic for long duration. There was no history of fever, haematemesis, malena, recent jaundice. No history of any major abdominal surgery in the past. Patient is not a known case of Diabetes and Hypertension (Figure 1).

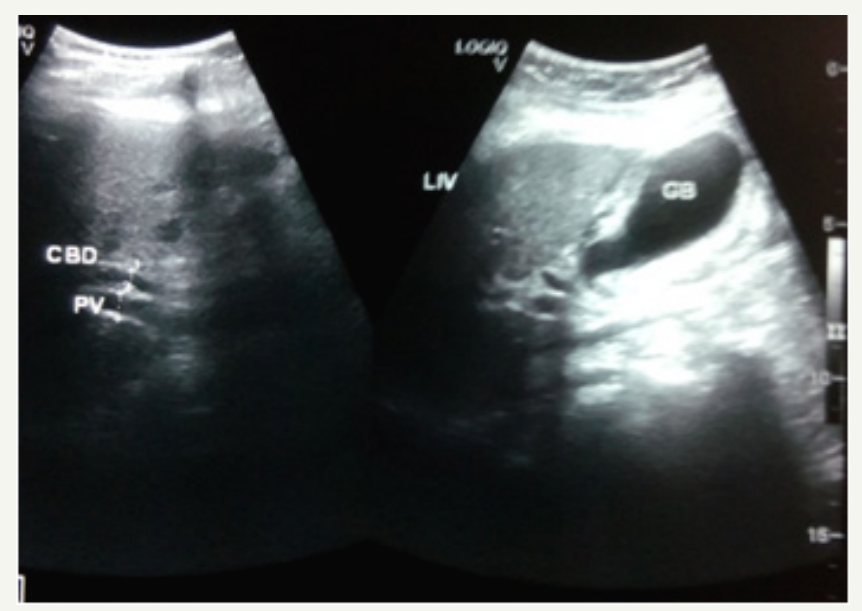

Figure 1: Pre-operative Ultrasonography Abdomen.

On clinical examination, Patient was alert, conscious, afebrile, dehydrated, pulse 68beats/minute, regular, Blood Pressure $160 / 90 \mathrm{~mm}$ of $\mathrm{Hg}$. On per abdominal examination, abdomen was mildly distended, epigastric tenderness with guarding was present. Intestinal peristaltic sounds were audible. No features of peritonitis 
were present. Per rectal examination was normal. Other systems were within normal limits.

Laboratory parameters on admission: Total count-8000/cu mm, Amylase 195IU/L, Lipase 217IU/L. Liver function tests revealed Total Bilirubin $2.30 \mathrm{mg} / \mathrm{dl}$, conjugated Bilirubin $1.5 \mathrm{mg} / \mathrm{dl}$, Alkaline Phosphatase 88IU/L, Electrolytes-serum Sodium 133meq/l, serum potassium $4.20 \mathrm{meq} / \mathrm{l}$. Ultrasound of the abdomen revealed normal Gall Bladder with hepatomegaly with fatty changes with mild free fluid in the abdomen. X-Ray Chest and Straight X-Ray Abdomen were Normal. Patient was started on nil per mouth with intravenous antibiotics and intravenous fluids. 1 day post admission, Computerized Tomography Scan of the Abdomen was done which revealed mural thickening at appendicular region with mild ascites and bilateral pleural effusion with basal segmental atelectasis (Figure 2). Laboratory parameters were Amylase 182IU/L, Lipase 274IU/L, Total Bilirubin $3.20 \mathrm{mg} / \mathrm{dl}$, conjugated Bilirubin $2.20 \mathrm{mg} / \mathrm{dl}$, Alkaline Phosphatase 78IU/L, Ionised Calcium $3.20 \mathrm{mg} / \mathrm{dl}$, serum Sodium $129 \mathrm{meq} / \mathrm{l}$, serum potassium $4.30 \mathrm{meq} / \mathrm{l}$. The patiemy developed increased abdominal distension, non passage of flatus and faeces, vomiting- non projectile, bilious, multiple episodes. Total leucocyte count was $7500 /$ cumm. Patient was haemodynamically unstable and was subsequently posted for Urgent exploratory laparotomy.

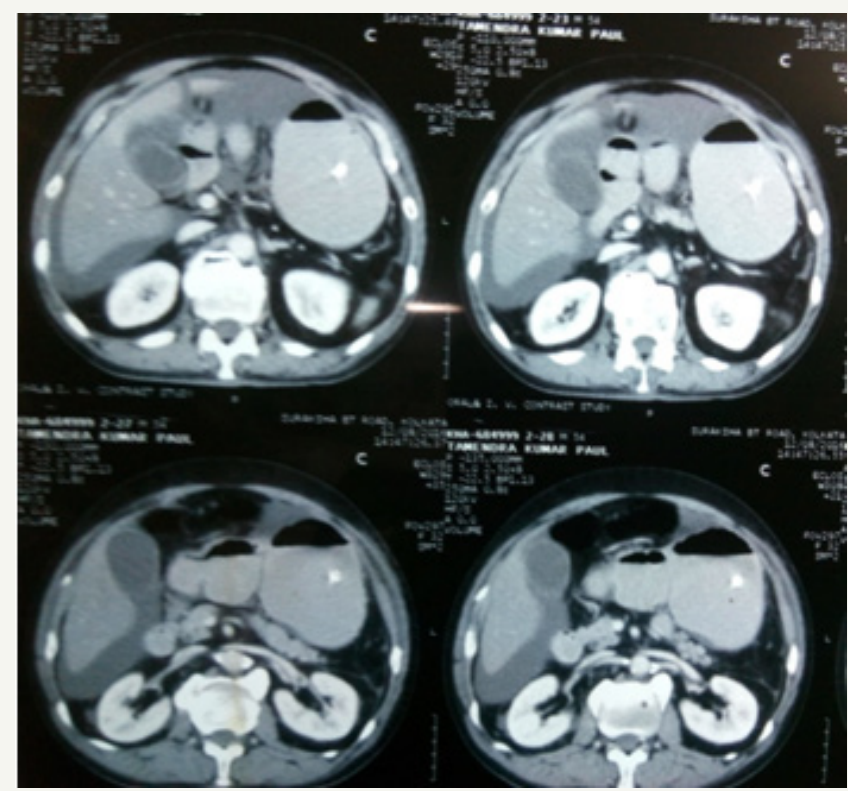

Figure 2: Pre-operative Computerized Tomography of Abdomen.

Abdomen opened by standard midline incision. Massive bile stained ascites approximately 4 litre was drained. Appendix, Caecum, other parts of small bowel and large bowel were found normal except a few flakes at the anterior wall of the stomach near the pylorus. Features suggestive of acalculus cholecystitis with spontaneous gall bladder wall perforation at the fundus were found (Figure $3 \& 4$ ). Cholecystectomy with thorough peritoneal toiletting was done. Drain was placed in the Historical pouch of Morrison. Abdomen was closed in single layer and the specimen of gall bladder was sent for histopathologial examination.

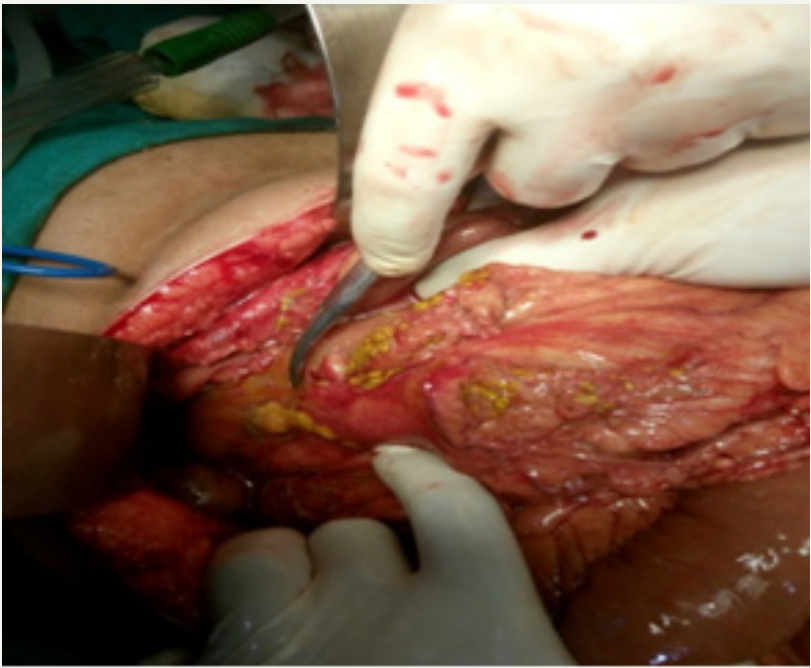

Figure 3: Intra-operative picture focussing on the perforation at the fundus of the Gall Bladder.

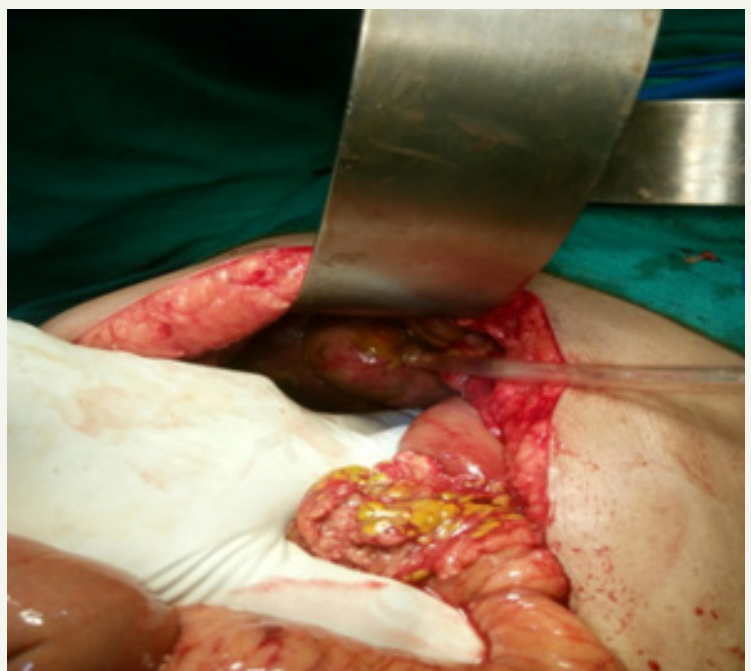

Figure 4: Intra-operative picture focusing on the perforation at the fundus of the Gall Bladder.

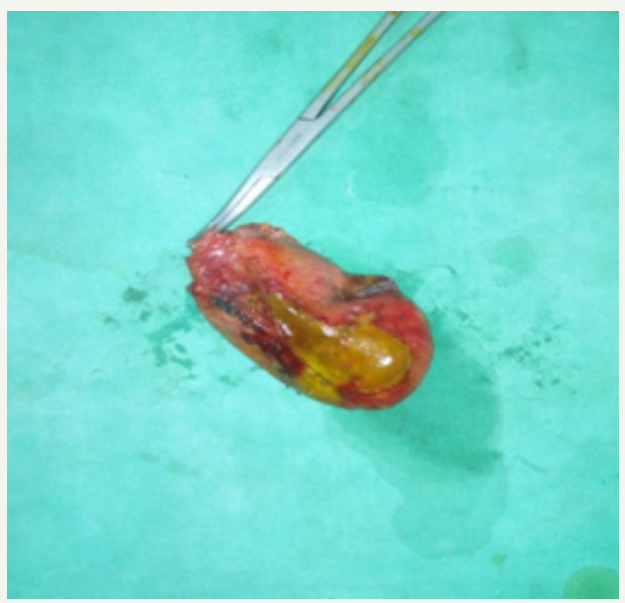

Figure 5: Post-operative specimen of Gall Bladder showing perforation at the fundus. 
Post operatively, the patient had an uncomplicated recovery. Drain was removed on $3^{\text {rd }}$ post operative day and the patient was discharged 1 week later after stitches were removed. Histopathological report revealed gall bladder perforation at the fundus on gross examination and ischaemic necrosis of gall bladder mucosa on microscopic examination (Figure 5).

\section{Discussion}

Even though there are many reports concerning gallbladder perforation, some controversy still remains regarding the tools of the early diagnosis and the therapeutic modalities [7]. Unlike gallbladder perforation, as a complication of cholecystitis, cases of gallbladder perforation without an apparent cause are rare and are reported vaguely as being either idiopathic or spontaneous. 5 Early diagnosis of gallbladder perforation and immediate surgical intervention are of prime importance in decreasing morbidity and mortality associated with this condition.

In this case, the exact diagnosis was elusive because of the scanty clues suggesting gallbladder perforation. The bile stained ascites, which led us to perform the cholecystectomy, was the only clue.

The presence of risk factors certainly warrants an aggressively oriented investigation stratagem to rule out this serious complication. However, it is also important to consider this condition as an important differential in patients without any prior features or history of gallstone disease but whose acute presentation may be indicative of biliary pathology as was the case in this patient.

Perforation can develop early in the course of acute cholecystitis (one or two days) or it may even occur several weeks after onset. The most common mechanism of gallbladder perforation involves cystic duct obstruction leading to gallbladder distension, vascular compromise, ischemia, necrosis, and ultimately perforation [8] Gallbladder perforation in the absence of gallbladder distension is likely related to infection of the Rokitansky-Aschoff sinuses with subsequent necrosis and rupture [9]. Because of its poor blood supply, the fundus of the gallbladder is the most common site of perforation. If the perforation locates at the fundus, it is less likely to be covered by the omentum thus bile and stones are likely to drain into the peritoneal space. If the perforation occurs at the isthmus or ductus, it is more easily sealed off by the omentum or the intestines and the condition remains limited to the right upper quadrant with formation of local inflammation and pericholecystic fluid.

Emphysematous, gangrenous, and hemorrhagic cholecystitis may progress to gallbladder perforation. Diagnosis is often difficult because the clinical symptoms of gallbladder perforation and variants of acute cholecystitis overlap. The high morbidity and mortality are attributed to delayed diagnosis and subsequent late surgical intervention. Gallbladder perforation is more common in men than in women and occurs at an average age of 60 years [10]. Since, there are no classical symptoms and signs of perforation diagnosis is challenging. Right upper quadrant pain, palpable right upper quadrant tenderness or high fever may indicate an acute onset. On the other hand patients may also show weakness, malaise and a palpable right upper quadrant mass, mimicking a malignancy. As most of these features are also present in acute cholecystitis, it is difficult to discriminate clinically between patients with perforated gallbladder and those with uncomplicated acute cholecystitis. A sudden decrease in pain intensity caused by the relief of high intracholecystic pressure might herald the perforation according to Chen et al. [11]. Gore et al. [12] suggested that perforation and abscess formation should be suspected in those patients with acute cholecystitis who suddenly become toxic and whose clinical condition is found to deteriorate rapidly [12].

Although ultrasound remains the preferred initial examination for evaluation of suspected gallbladder perforation, unfortunately it often fails to demonstrate the perforation because of increased intestinal gas and pain. In contrast, CT imaging is the most sensitive tool to diagnose gallbladder perforation $[13,14]$.

In our case, both Ultrasonography as well as CT scan failed to throw any light on the diagnosis. The reports were essentially nonspecific. Post exploratory laparotomy with cholecystectomy with thorough peritoneal lavage, the biliary peritonitis resolved and the patient recovered well (Figure 1).

Therefore, it is believed that this is a case of spontaneous gallbladder perforation, probably due to an ischemic cause. Therefore, clinicians should take this disorder into consideration when encountering cases of elderly patients presenting with an acute abdomen with an unknown origin. In conclusion gallbladder perforation is a rare but very serious condition and should be diagnosed and treated as soon as possible to decrease morbidity and mortality.

\section{Consent/Ethical approval}

Written informed consent was obtained from the patient for publication of this article and accompanying images. A copy of the written consent is available for review by the journal's Editor-inchief.

\section{References}

1. Roslyn J, Busuttil RW (1979) Perforation of the gallbladder: a frequently mismanaged condition. Am J Surg 137(3): 307-312.

2. Derici H, Kara C, Bozdag AD, Nazli O, Tansug T, et al. (2006) Diagnosis and treatment of gallbladder perforation. World J Gastroenterol 12(48): 7832-7836.

3. Anderson BB, Nazem A (1987) Perforations of the gallbladder and cholecystobiliary fistulae: a review of management and a new classification. J Natl Med Assoc 79(4): 393-399.

4. Bakalakos EA, Melvin WS, Kirkpatrick R (1996) Liver abscess secondary to intrahepatic perforation of the gallbladder, presenting as a liver mass. Am J Gastroenterol 91(8): 1644-1646.

5. Tanaka M, Takahashi H, Yajima Y, Okamura K, et al. (1997) Idiopathic perforation of the gallbladder report of a case and a review of the Japanese literature. Surg Today 27(4): 360-363.

6. Estevao-Costa J, Soares-Oliveira M, Lopes JM, Carvalho JL (2002) Idiopathic perforation of the gallbladder: a novel differential diagnosis of acute abdomen. J Pediatr Gastroenterol Nutr 35(1): 88-89.

7. Kwon KH, Hong SJ, Park CW, Song DH, Lee JS, et al. (1994) A case of gallbladder perforation treated by percutaneous transhepatic 
chotecystic drainage and percutaneous peritoneal drainage. Korean Gastrointesti Endosc 14: 482-488.

8. zo BL, Francis I, Hricak H, Sandler MA, Hudak S, et al. (1982) Sonographic findings in perforation of the gallbladder. AJR Am J Roentgenol 139(3): 491-496.

9. Morris BS, Balpande PR, Morani AC, Chaudhary RK, Maheshwari M, et al. (2007) The CT appearances of gallbladder perforation. $\mathrm{Br}$ Radiol 80(959): 898-901.

10. Stefanidis D, Sirinek KR, Bingener J (2006) Gallbladder perforation: risk factors and outcome. J Surg Res 131(2): 204-208.

11. Chen JJ, Lin HH, Chiu CT, Lin DY (1990) Gallbladder perforation with intrahepatic abscess formation. J Clin Ultrasound 18(1): 43-45.

12. Gore RM, Ghahremani GG, Joseph AE, Nemcek AA, Marn CS, et al. (1989) Acquired malposition of the colon and gallbladder in patients with cirrhosis: CT findings and clinical implications. Radiology 171: 739-742.

13. Sood BP, Kalra N, Gupta S, Sidhu R, Gulati M, et al. (2002) Role of sonography in the diagnosis of gallbladder perforation. J Clin Ultrasound 30: $270-274$.

14. Kochar K, Vallance K, Mathew G, Jadhav V (2008) Intrahepatic perforation of the gall bladder presenting as liver abscess: case report, review of literature and Niemeier's classification. Eur J Gastroenterol Hepatol. 2008, 20: 240-244. 